\title{
Plantas medicinais: cultivo em quintais pela população de um município do semiárido Piauiense, nordeste do Brasil
}

\author{
Maria de Fátima Pereira da Silva \\ Licenciada em Ciências Biológicas, Universidade Federal do Piauí. \\ Campus CSHNB.
}

\section{Paulo Henrique da Silva}

Licenciado em Ciências Biológicas, Universidade Federal do Piauí. Campus CSHNB.

\section{Ykaro Richard Oliveira}

Licenciado em Ciências Biológicas, Universidade Federal do Piauí. Campus CSHNB.

\section{Tássio Marcílio Francisco Gomes}

Especialista em Educação Ambiental, Professor Auxiliar I, Curso de Ciências Biológicas, Universidade Federal do Piauí. Campus Senador Helvídio Nunes de Barros.

\section{Paulo Michel Pinheiro Ferreira}

Doutor em Farmacologia, Programa de Pós-graduação em Ciências Farmacêuticas, Departamento de Biofísica e Fisiologia, Centro de Ciências da Saúde, Universidade Federal do Piauí.

\section{Gilberto Santos Cerqueira}

Pós-doutor em Morfologia, Programa de Pós-Graduação em Ciências Farmacêuticas da Universidade Federal do Piauí. Laboratório de Anatomia Campus CSHNB.

SILVA, Maria de Fátima Pereira da; SILVA, Paulo Henrique da; OLIVEIRA, Ykaro Richard; GOMES, Tássio Marcílio Francisco; FERREIRA, Paulo Michel Pinheiro; CERQUEIRA, Gilberto Santos; ABREU, Maria Carolina de. Plantas medicinais: cultivo em quintais pela população de um município do semiárido Piauiense, nordeste do Brasil. RevInter Revista Intertox de Toxicologia, Risco Ambiental e Sociedade, v. 7, n. 3, p. 101113, out. 2014. 


\section{Maria Carolina de Abreu}

Doutora em Botânica, Professora Adjunto II, Curso de Ciências Biológicas, Universidade Federal do Piauí- Campus Senador Helvídio Nunes de Barros. E-mail: mariacarolinabreu@hotmail.com.

\section{RESUMO}

O uso das plantas medicinais cultivadas em quintais, é uma prática recorrente entre populações de todo planeta, por questões socioeconômicas ou culturais, tanto em zonas rurais como urbanas. É evidente a importância dos quintais facilitando as ações antropogênicas e condicionando a manutenção desses recursos vegetais no período de estiagem. Esse estudo objetiva levantar dados no que concerne o cultivo e uso de plantas medicinais nos quintais. Ele foi realizado na cidade de São Julião, no semiárido Piauiense. Estudo de caráter quali-quantitativo descritivo, realizado entre fevereiro e maio de 2013, sendo considerado o Termo de Consentimento Livre e Esclarecido, além de aplicar 100 entrevistas semiestruturadas, onde as residências foram escolhidas aleatoriamente. Registrou-se o uso e o cultivo de oito espécies, distribuídas em 06 famílias, sendo a Lamiaceae (03) de maior ocorrência. Constatou-se o cultivo em quintais em $24 \%$ das citações, e o uso destas em 91,67\%. Das partes utilizadas predominou a folha com $83,33 \%$, e como forma de preparo foi a infusão com $79,17 \%$. Com base no exposto, o pequeno número de plantas medicinais cultivadas em quintais, leva a indagações e contradições de cunho cultural e ético, carecendo de mais estudos na região para compreender os motivos do abandono destas práticas.

Palavras - chave: Conhecimento popular, Caatinga, Cultivo e uso de plantas.

SILVA, Maria de Fátima Pereira da; SILVA, Paulo Henrique da; OLIVEIRA, Ykaro Richard; GOMES, Tássio Marcílio Francisco; FERREIRA, Paulo Michel Pinheiro; CERQUEIRA, Gilberto Santos; ABREU, Maria Carolina de. Plantas medicinais: cultivo em quintais pela população de um município do semiárido Piauiense, nordeste do Brasil. RevInter Revista Intertox de Toxicologia, Risco Ambiental e Sociedade, v. 7, n. 3, p. 101113, out. 2014. 


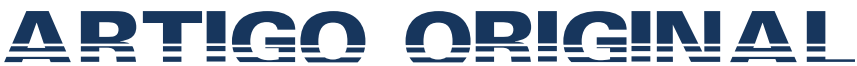

\section{ABSTRACT}

The use of medicinal plants cultivated in backyards is an applicant practice among peoples across the planet by socioeconomic or cultural issues in rural and urban areas. The importance of backyards is evident, facilitating the anthropogenic actions and conditioning the maintenance of these plant resources in drought. The study aim was to get information about cultivation and use of medicinal plants in backyards, at São Julião city in semi-arid of Piauí state, Brazil. The study has a quali-quantitative approach with descriptive character and was conducted between February and May 2013, was been considered the informed consent for research, with 100 semistructured interviews applied and households randomly selected. Was recorded the use and cultivation of eight species, distributed in 06 families, being the Lamiaceae (03) family of higher occurrence. Cultivation in backyards occurred in $24 \%$ of the citations, and the use of these in $91.67 \%$. The most used part of the plants was the leaf $83.33 \%$, and the infusion was the method of preparation most common 79.17\%. Based on the exposed, the small number of medicinal plants cultivated in backyards leads to questions and contradictions with cultural and ethical character, needing more studies to understand the abandonment of these practices.

Keywords: Popular knowledge, Caatinga, Cultivation and use of plants.

SILVA, Maria de Fátima Pereira da; SILVA, Paulo Henrique da; OLIVEIRA, Ykaro Richard; GOMES, Tássio Marcílio Francisco; FERREIRA, Paulo Michel Pinheiro; CERQUEIRA, Gilberto Santos; ABREU, Maria Carolina de. Plantas medicinais: cultivo em quintais pela população de um município do semiárido Piauiense, nordeste do Brasil. RevInter Revista Intertox de Toxicologia, Risco Ambiental e Sociedade, v. 7, n. 3, p. 101113, out. 2014. 


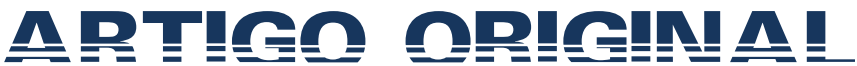

\section{INTRODUÇÃO}

Considerando as palavras de Salvi e Heuser (2008), planta medicinal é todo e qualquer vegetal que uma vez aplicado sob determinada maneira e por alguma via ao ser humano é capaz de promover um efeito farmacológico. A utilização de plantas medicinais é uma prática comum entre as populações. Cerca de $80 \%$ da população mundial depende da medicina tradicional para suas necessidades básicas de saúde e, quase $85 \%$ da medicina tradicional envolve o uso de plantas medicinais, seus extratos vegetais e seus princípios ativos (OMS 2002). Em consonância com Lima e colaboradores (2010), acrescenta-se que a utilização de plantas medicinais tem incentivos inclusive da Organização Mundial da Saúde.

Estes vegetais têm um papel muito importante na questão socioeconômica, tanto para as populações que vivem em meio rural quanto para as que vivem no meio urbano. A utilização de espécies medicinais, na maioria das vezes nativa da sua região, ou cultivadas em quintal, pode reduzir os gastos com medicamentos alopáticos (CALIXTO 2000). Por outro lado, Di Stasi (2007) reporta que o avanço da ciência e da tecnologia no que tange à elaboração sintética de fármacos vem desestimulando, ao longo do tempo, a utilização das plantas medicinais no cuidado à saúde.

Os quintais são considerados sistemas agroflorestais importantes para o cultivo, seleção e conservação de espécies de plantas, fornecendo recursos para o consumo familiar e para preservação da cultura local (KUMAR e NAIR 2004), sendo também apontados como responsáveis em conservar parte da história da cultural local, podendo ser considerados reservas atuais e potenciais de recursos vegetais (BRASIL et al. 2007).

SILVA, Maria de Fátima Pereira da; SILVA, Paulo Henrique da; OLIVEIRA, Ykaro Richard; GOMES, Tássio Marcílio Francisco; FERREIRA, Paulo Michel Pinheiro; CERQUEIRA, Gilberto Santos; ABREU, Maria Carolina de. Plantas medicinais: cultivo em quintais pela população de um município do semiárido Piauiense, nordeste do Brasil. RevInter Revista Intertox de Toxicologia, Risco Ambiental e Sociedade, v. 7, n. 3, p. 101113, out. 2014. 


\section{凡}

Desta forma, este espaço é de grande relevância para o contato com variadas espécies vegetais, bem como à manutenção de conhecimentos populares (NASCIMENTO et al. 2006; SIVIERO et al. 2011).

$\mathrm{Na}$ visão de Rocha e colaboradores (2010), os quintais facilitam as ações antropogênicas, principalmente no que se refere a procriar condições de sobrevivência nos períodos de seca do sertão nordestino, onde algumas plantas perdem suas folhas e outras chegam a morrer. Para Amorozo (2002) o hábito de cultivar plantas em quintais nas zonas urbanas permite formar através da união de vários espaços plantados, como praças e parques, ilhas de vegetação que contribuem para a melhoria da qualidade de vida nos centros urbanos.

É importante ressaltar que os quintais são compreendidos como um sistema de produção complementar a outras formas de uso da terra, podendo se destaca pelo valor econômico que desempenham na residência, constituindo uma fonte disponível de recursos alimentícios e medicinais (PASA, SOARES e GUARIM-NETO 2005).

Este trabalho teve como objetivo realizar um levantamento de dados sobre o cultivo e uso de plantas medicinais nos quintais da população do município de São Julião/PI.

\section{METODOLOGIA}

O levantamento foi realizado na cidade de São Julião, município piauiense de aproximadamente 5.761 habitantes (IBGE 2010), o qual foi fundado em 18 de dezembro de 1960 e dista $382 \mathrm{~km}$ da capital Teresina 382 $\mathrm{km}$.

O município dispõe de abastecimento de água, energia elétrica distribuída pela Companhia Energética do Piauí S/A - CEPISA, terminais

SILVA, Maria de Fátima Pereira da; SILVA, Paulo Henrique da; OLIVEIRA, Ykaro Richard; GOMES, Tássio Marcílio Francisco; FERREIRA, Paulo Michel Pinheiro; CERQUEIRA, Gilberto Santos; ABREU, Maria Carolina de. Plantas medicinais: cultivo em quintais pela população de um município do semiárido Piauiense, nordeste do Brasil. RevInter Revista Intertox de Toxicologia, Risco Ambiental e Sociedade, v. 7, n. 3, p. 101113, out. 2014. 


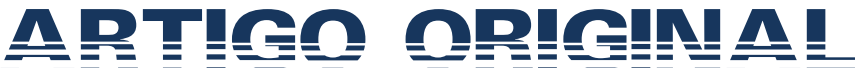

telefônicos atendidos pela TELEMAR Norte Leste S/A, agência de Correios e Telégrafos, hospital e escolas de ensino fundamental e médio. A agricultura praticada no município é baseada na produção sazonal de feijão, algodão, mandioca e milho.

No tocante às condições climáticas do município, ocorre o clima semiárido quente e seco, com temperaturas mínimas de $24^{\circ} \mathrm{C}$ e máximas de $36^{\circ} \mathrm{C}$. Localizado na altitude de $377 \mathrm{~m}$, apresenta solos, em grande parte provenientes da alteração de gnaisses, quartzito, mármore, xisto, arenito, siltito e conglomerado, rasos ou pouco espessos, jovens, às vezes pedregosos, ainda com influência do material subjacente (JACOMINE et al. 1986).

A coleta de dados foi realizada entre os habitantes do município de São Julião - PI, onde foram aplicados 100 formulários em residências escolhidas de forma aleatória nas zonas rural e urbana, no período de fevereiro a maio de 2013. O formulário semiestruturado foi composto por 14 questões abordando dados sobre o perfil dos participantes e questões sobre o hábito destes em utilizar e cultivar plantas medicinais em seus quintais. No processo de coleta de dados foram levadas em consideração as exigências contidas na Resolução 466/12 do Conselho Nacional de Saúde, que regulamenta a pesquisa com seres humanos (BRASIL 2012). Para tanto, os participantes assinaram o Termo de Consentimento Livre e Esclarecido assegurando aos participantes o anonimato, a privacidade e o direito de desistir em qualquer etapa da pesquisa. Ressalta-se que esse estudo não possui nenhum conflito de interesse, se constituindo numa pesquisa de caráter quali-quantitativa de natureza descritiva. Todos os formulários foram analisados à luz da literatura pertinente, e os dados compilados e os gráficos construídos por meio do programa Microsoft Excel 2010.

SILVA, Maria de Fátima Pereira da; SILVA, Paulo Henrique da; OLIVEIRA, Ykaro Richard; GOMES, Tássio Marcílio Francisco; FERREIRA, Paulo Michel Pinheiro; CERQUEIRA, Gilberto Santos; ABREU, Maria Carolina de. Plantas medicinais: cultivo em quintais pela população de um município do semiárido Piauiense, nordeste do Brasil. RevInter Revista Intertox de Toxicologia, Risco Ambiental e Sociedade, v. 7, n. 3, p. 101113, out. 2014. 


\section{凡}

\section{RESULTADOS E DISCUSSÃO}

Os dados obtidos através das 100 entrevistas, norteadas pelo formulário de 14 questões aplicados à população do município de São JuliãoPI, permitiram estabelecer o perfil da população deste município quanto ao cultivo de plantas medicinais em quintais além da diversidade e uso destas plantas.

Quanto ao gênero dos participantes, $26 \%$ foram do sexo masculino e $74 \%$ do sexo feminino. Em um estudo semelhante de Nascimento e Gonçalves (2013) para avaliar o uso de plantas cultivadas em quintais urbanos no município de Campina do Monte Alegre em São Paulo, também prevaleceu o maior conhecimento entre as mulheres que parece estar mais voltado ao cultivo e a utilização de plantas medicinais.

O grau de escolaridade dos entrevistados variou entre Ensino Fundamental incompleto (39\%), Ensino Fundamental completo (22\%), Ensino Médio incompleto (8\%), Ensino Médio completo (17\%), Graduação incompleta (4\%), Graduação completa (8\%) e Pós-graduação (2\%). Percebe-se uma maioria de sujeitos com ensino fundamental incompleto, o que denota uma baixa escolaridade dos envolvidos no estudo. Todavia, ter baixa escolaridade não significa que o indivíduo não conhece sobre o cultivo e a utilização de determinadas plantas, pois tal saber pode ter sido a ele repassado de forma oral pelos familiares e na comunidade.

Em uma pesquisa semelhante sobre plantas venenosas de Silva e colaboradores (2014) na cidade de Francisco Santos, que fica à $60 \mathrm{~km}$ de São Julião, também prevaleceu a escolaridade de Ensino Fundamental incompleto. Porém, um estudo sobre a ocorrência do uso de plantas medicinais por moradores de um bairro do município de Marília-SP, mostra que $31 \%$ dos participantes possuía nível superior completo, levando-nos a

SILVA, Maria de Fátima Pereira da; SILVA, Paulo Henrique da; OLIVEIRA, Ykaro Richard; GOMES, Tássio Marcílio Francisco; FERREIRA, Paulo Michel Pinheiro; CERQUEIRA, Gilberto Santos; ABREU, Maria Carolina de. Plantas medicinais: cultivo em quintais pela população de um município do semiárido Piauiense, nordeste do Brasil. RevInter Revista Intertox de Toxicologia, Risco Ambiental e Sociedade, v. 7, n. 3, p. 101113, out. 2014. 


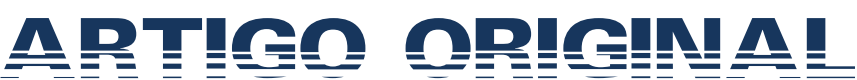

acreditar que o cultivo e o consumo de plantas medicinais não se dá apenas em classes de condições socioeconômicas menos favorecidas.

No que se refere à faixa etária dos entrevistados, há uma maior frequência na classe de idade compreendida entre 31 a 55 anos (38\%). Em seguida, com um número muito próximo de entrevistados (36\%) aparece a faixa etária de 18 a 30 anos. A menor frequência foi a da faixa etária entre 76-90 anos com 3\%. Silva e colaboradores (2014) aponta a faixa etária de 7690 como sendo dotada de maior conhecimento sobre os recursos naturais, por terem sentido ao longo da vida a necessidade de recorrer às plantas como fonte de medicamento.

Sobre a renda mensal familiar dos entrevistados, prevaleceu a renda média de um salário mínimo (55\%), sendo relevante também informar a percentagem de pessoas com renda abaixo de um salário mínimo (43\%).

Desta forma, através dos dados obtidos na pesquisa, constatou-se que o perfil da amostra questionada é de mulheres de baixa escolaridade com a faixa etária entre 31 a 55 anos, com renda familiar de um salário mínimo.

Do universo de entrevistados, apenas $24 \%$ cultivam plantas medicinais em seus quintais e os demais 76\% não cultivam. Assim, é interessante ressaltar a pequena quantidade de pessoas que cultivam plantas medicinais.

Considerando os indivíduos que ocorrem na porcentagem dos que cultivam (24\%), apenas oito espécies foram mencionadas por estes, as quais são cultivadas e usadas para finalidades medicinais, conforme evidencia-se na Tabela 1.

Tabela 1. Nomes vulgares, nomes científicos e famílias das plantas medicinais cultivadas nos quintais pela população do município de São Julião - PI.

SILVA, Maria de Fátima Pereira da; SILVA, Paulo Henrique da; OLIVEIRA, Ykaro Richard; GOMES, Tássio Marcílio Francisco; FERREIRA, Paulo Michel Pinheiro; CERQUEIRA, Gilberto Santos; ABREU, Maria Carolina de. Plantas medicinais: cultivo em quintais pela população de um município do semiárido Piauiense, nordeste do Brasil. RevInter Revista Intertox de Toxicologia, Risco Ambiental e Sociedade, v. 7, n. 3, p. 101113, out. 2014. 


\begin{tabular}{lc}
\hline FAMILIA/ESPECIE & NOME VULGAR \\
\hline AMARANTHACEAE & Mastruz \\
Chenopodium ambrosioides L. & \\
LAMIIACEAE & Hortelã \\
Mentha spicata L. & malva-do-reino \\
Plectranthus amboinicus (Lour.) Spreng. & Boldo \\
Plectranthus barbatus Andrews & \\
LYTHRACEAE & Romã \\
Punica granatum L. & \\
POACEAE & capim-santo \\
Cymbopogon citratus (DC.) Stapf & \\
RUTACEAE & Arruda \\
Ruta graveolens L. & \\
VERBENACEAE & \\
Lippia alba (Mill.) N.E. Br. ex Britton \& P. Wilson & erva-cidreira \\
\hline
\end{tabular}

Em relação ao uso das plantas cultivadas, indagou-se aos envolvidos na pesquisa se quando utilizam as plantas medicinais, fazem uso daquelas que cultivam ou buscam em outros lugares. Para tal questão, $22 \%$ das pessoas questionadas faz uso daquelas que cultivam e, $78 \%$ busca em outros lugares.

Da parcela de indivíduos que cultiva plantas medicinais em seus quintais (24), 91,67\% (22) faz uso daquelas que cultivam. Com relação ao local em que têm acesso às plantas medicinais, a grande maioria (58\%) mencionou o mercado, $39 \%$ citou o acesso com vizinhos e apenas $3 \%$ fez referência à mata.

Esses dados mostram que, apesar de a maioria ter acesso no mercado, ainda existe um grande compartilhamento de plantas entre vizinhos. E mesmo não cultivando plantas medicinais, as pessoas as utilizam muito para fins terapêuticos. Somavilla e Canto-Dorow (1996) verificaram que 76\% das plantas utilizadas como medicinais na cidade de Santa Maria (RS) são obtidas através de amigos e também pelo hábito de cultivo caseiro.

Aos que afirmaram cultivar plantas em seus quintais, foi questionado se fazem uso de algum tipo de adubo e também se utilizam agrotóxico. As 24

SILVA, Maria de Fátima Pereira da; SILVA, Paulo Henrique da; OLIVEIRA, Ykaro Richard; GOMES, Tássio Marcílio Francisco; FERREIRA, Paulo Michel Pinheiro; CERQUEIRA, Gilberto Santos; ABREU, Maria Carolina de. Plantas medicinais: cultivo em quintais pela população de um município do semiárido Piauiense, nordeste do Brasil. RevInter Revista Intertox de Toxicologia, Risco Ambiental e Sociedade, v. 7, n. 3, p. 101113, out. 2014. 


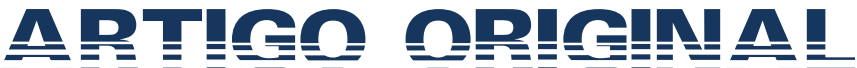

pessoas que cultivam plantas utilizam o adubo orgânico e 95,83 \% (23) não usam nenhum tipo de agrotóxico, apenas 4,17\% (1) faz uso de veneno para matar pragas.

Com relação a utilização das plantas medicinais cultivadas, 83,33\% (20) dos entrevistados que cultivam plantas medicinais em seus quintais (24) faz corte na planta utilizando apenas as folhas. Pereira-Martins (2001) na comunidade negra de Abacatal-PA, também teve as folhas (57\%) como a parte da planta mais indicada. Os demais $16,67 \%$ (4) não faz nenhum corte e utiliza toda a planta. Já a forma de preparo predominante na comunidade é a infusão, mencionada por $79,17 \%$ (19) dos sujeitos que cultivam plantas medicinais no município de São Julião. A infusão é um tipo de chá que é ingerido de forma oral.

$\mathrm{Na}$ população de São Julião esses chás são as preparações terapêuticas mais populares, sendo usados para quase todas as indicações de cura e prevenção das doenças, como por exemplo, tosse, gripes, diarreias, problemas de fígado, intestinos, calmante, diurético, cólicas, insônia e dores em geral. Outro tipo de preparo utilizado é o lambedor, citado por 20,83\% (5) da população que cultiva plantas medicinais e é utilizado para dores de garganta e no tratamento de gripes.

Outro dado que investigou-se refere-se a época do ano que os entrevistados mais utilizam remédios caseiros preparados a partir das plantas cultivadas. A maioria deles $71 \%$ não fazem diferença do uso, $17 \%$ utilizam mais no período chuvoso (janeiro a maio) e $12 \%$ no período seco (junho a dezembro). Resultados semelhantes foram obtidos por Pereira (2010) em uma pesquisa sobre levantamento de dados sobre o uso e os costumes da utilização de plantas medicinais pela população picoense, no qual a grande maioria dos entrevistados (69,5 \%) também não faziam diferença entre os períodos seco e chuvoso no uso das plantas medicinais.

SILVA, Maria de Fátima Pereira da; SILVA, Paulo Henrique da; OLIVEIRA, Ykaro Richard; GOMES, Tássio Marcílio Francisco; FERREIRA, Paulo Michel Pinheiro; CERQUEIRA, Gilberto Santos; ABREU, Maria Carolina de. Plantas medicinais: cultivo em quintais pela população de um município do semiárido Piauiense, nordeste do Brasil. RevInter Revista Intertox de Toxicologia, Risco Ambiental e Sociedade, v. 7, n. 3, p. 101113, out. 2014. 


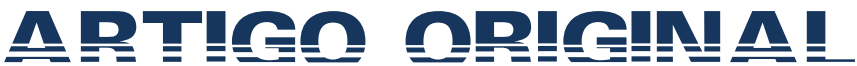

\section{CONSIDERAÇÕES FINAIS}

Em decorrência dos resultados analisados, é perceptível a pequena quantidade de plantas medicinais que são cultivadas nos quintais da região estudada. Seria a explicação para tal fato o grande desenvolvimento da ciência e da farmacologia no que se refere o desenvolvimento de medicamentos alopáticos e homeopáticos e a facilidade de adquiri-los? Ou a descrença da população investigada quanto às propriedades bioativas que os vegetais apresentam e que podem proporcionar benefícios na terapia de inúmeras doenças? Ou seria a questão da mudança de padrões e de hábitos quanto o cultivo de plantas medicinais na região de São Julião-PI, que já sofre as pressões decorrentes da modernidade?

São respostas estas, necessárias para compreender o panorama seja cultural seja ético em que tal localidade está inserida, haja vista que o uso e cultivo de plantas está inserido na cultura popular, vinculados à disseminação do conhecimento referente a tais ao longo das gerações. Assim, carecem estudos para melhor compreender os motivos que estão levando a população em foco a abandonar às práticas de cultivo das plantas medicinais em quintais locais.

\section{REFERÊNCIAS BIBLIOGRÁFICAS}

AMOROSO, M. C. M. Uso e diversidade de plantas medicinais em Santo Antônio do Leverger, MT, Brasil. Acta Botânica Brasílica, v. 1, p. 189-203, 2002.

BRASIL, R. D.; SOLDATI, G. T.; COSAT, F.V.; MARCATTI, A.A; JR, R.R.; COELHO, F.M.G . 2007. Riqueza de Plantas e Estrutura de Quintais Familiares no Semi-árido Norte Mineiro. Revista Brasileira de Biociências, Porto Alegre, v. 5, p. 864-866.

BRASIL. Ministério da Saúde. Conselho Nacional de Saúde. Comissão Nacional de Ética em Pesquisa. Normas para pesquisa envolvendo seres humanos: (Res. CNS 466/12 e outros) Brasília, DF, 2012. Acesso em: 19/09/2014.

SILVA, Maria de Fátima Pereira da; SILVA, Paulo Henrique da; OLIVEIRA, Ykaro Richard; GOMES, Tássio Marcílio Francisco; FERREIRA, Paulo Michel Pinheiro; CERQUEIRA, Gilberto Santos; ABREU, Maria Carolina de. Plantas medicinais: cultivo em quintais pela população de um município do semiárido Piauiense, nordeste do Brasil. RevInter Revista Intertox de Toxicologia, Risco Ambiental e Sociedade, v. 7, n. 3, p. 101113, out. 2014. 


\section{凡}

CALIXTO, J. B. Efficacy, safety, quality control, marketing and regulatory guidelines for herbal medicines (phytotherapeutic agents). Braz. J. Med. Biol. Res.,v. 33, n. 2, p. 79-89, 2000.

DI STASI, L. C. Plantas Medicinais: verdades e mentiras: o que os usuários e os profissionais de saúde precisam saber. São Paulo: UNESP; 2007.

Instituto Brasileiro de Geografia e Estatística (IBGE). Resultados do censo 2010. Disponível em: <http://www.ibge.gov.br/censo2010>.

JACOMINE, P.K.T. et al. Levantamento exploratório - reconhecimento de solos do Estado do Piauí. Rio de Janeiro. EMBRAPA-SNLCS/SUDENE-DRN. 1986. 782 p.

KUMAR, B.M.; NAIR, P.K.R. the enigma of tropical home gardens. Agroforestry systens, vol. 19, p. 195-207, 2004.

LIMA, J.F; SILVA, M.P.L.; TELES, S.; SILVA, F.; MARTINS, G. N. Avaliação de diferentes substratos na qualidade fisiológica de sementes de melão de caroá [Sicana odorifera (Vell.) Naudim]. Revista Brasileira de Plantas Medicinai, v. 12, n.2, p.163-167, 2010.

MACEDO, A.F; OSHIIWA, M.; GUARIDO, C.F.Ocorrência do uso de plantas medicinais por moradores de um bairro do município de Marília-SP. Rev. Ciênc. Farm. Básica Apl. v. 28, n.1, p.123-128, 2006.

NASCIMENTO, A. P. L.F; GONÇALVES, K. (2013).Uso de plantas cultivadas em quintais urbanos no município de campina do Monte Alegre, SP. XI Congresso de Ecologia do Brasil, Setembro 2013, Porto Seguro - BA.

NASCIMENTO, A.P.B.; SILVA, M.R.; GARAVELLO, M.E.P.E.; MOLINA, S.M.G. Quintais domésticos: conhecimento, tradição e utilidades no município de Piracicaba (SP). In: EHWC'2006 Environmental and Health World Congress, Santos.

ORGANIZACIÓN MUNDIAL DE LA SALUD (OMS). Estrategia de la OMS sobre medicina tradicional 2002-2005. Genebra, Switzerland, 2002.

PASA, M.C. SOARES, J.N e GUARIM-NETO,G. Estudo etnobotanico na comunidade de Conceição- Açu (alto da bacia do rio Aricá Açu, MT, Brasil). Acta Botânica Brasílica, v. 19, p. 195-207, 2005.

PEREIRA, J. B.A. Levantamento de dados sobre o uso e os costumes da utilização de plantas medicinais pela população picoense $44 p$. (Monografia Graduaçãoem Licenciatura Ciências Biológicas)-Departamento de Biologia, Universidade Federal d Piauí, Picos -PI, 2010.

PEREIRA-MARTINS, N. C. Abordagem etnobotânica de plantas medicinais e alimentícias na comunidade negra de Abacatal, Ananindeua - PA. Dissertação (Mestrado em Agronomia) Departamento de Biologia Vegetal, Faculdade de Ciências Agrárias do Pará, Belém, 138 f. 2001.

SILVA, Maria de Fátima Pereira da; SILVA, Paulo Henrique da; OLIVEIRA, Ykaro Richard; GOMES, Tássio Marcílio Francisco; FERREIRA, Paulo Michel Pinheiro; CERQUEIRA, Gilberto Santos; ABREU, Maria Carolina de. Plantas medicinais: cultivo em quintais pela população de um município do semiárido Piauiense, nordeste do Brasil. RevInter Revista Intertox de Toxicologia, Risco Ambiental e Sociedade, v. 7, n. 3, p. 101113, out. 2014. 


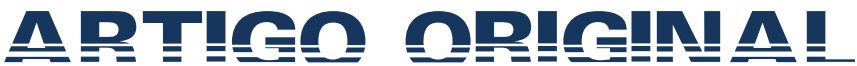

ROCHA, V.M.S; GOMES, T.M.F; LEAL, C.B; ABREU, M.C; SANTOS, L.L. A cultura de Preparo e Partes Usadas das Plantas da Caatinga, na Medicina Popular: dados preliminares. III Encontro de Ciências Biológicas, II Jornada de Iniciação Cientifica. UFPI, Campus Senador Helvídio Nunes de Barros. 2010

SALVI, R. M. e HEUSER, E. D. Interações medicamentos x fitoterápicos: em busca de uma prescrição racional. Porto Alegre: EDIPUCRS, 2008. 116p.

SILVA, L.R. R; ABREU, M.C.; FERREIRA, P. M. P; PACHECO, A. C. L.; CALOU, I. B. F.; CERQUEIRA, G. S. Plantas Tóxicas: conhecimento de populares para prevenção de acidentes. Revinter Revista Intertox de Toxicologia, risco Ambiental e Sociedade, v. 7, n. 2, p. 17-36, 2014.

SIVIERO, A., DELUNARDO, T.A., HAVERROTH, M., OLIVEIRA, L.C., MENDONÇA, A.M.S. Cultivo de Espécies Alimentares em Quintais Urbanos de Rio Branco, Acre, Brasil. Acta Botanica Brasilica. v.25, n.3, p.549-556, 2011.

SOMAVILLA N, CANTO-DOROW TS. Levantamento das plantas medicinais utilizadas em bairros de Santa Maria, RS, Brasil. Cienc Natura v. 18, p. 31-148,1996.

SILVA, Maria de Fátima Pereira da; SILVA, Paulo Henrique da; OLIVEIRA, Ykaro Richard; GOMES, Tássio Marcílio Francisco; FERREIRA, Paulo Michel Pinheiro; CERQUEIRA, Gilberto Santos; ABREU, Maria Carolina de. Plantas medicinais: cultivo em quintais pela população de um município do semiárido Piauiense, nordeste do Brasil. RevInter Revista Intertox de Toxicologia, Risco Ambiental e Sociedade, v. 7, n. 3, p. 101113, out. 2014. 